\title{
Impact de différents scénarios énergétiques sur les matières premières et leur disponibilité future
}

\author{
Par Olivier VIDAL \\ Institut des sciences de la terre (ISTerre), Université Grenoble Alpes, CNRS
}

\begin{abstract}
La population mondiale, le PIB moyen/hab, ainsi que la consommation d'énergie et de matières premières affichent les mêmes croissances quasi exponentielles depuis plus d'un siècle. L'humanité utilise désormais des ressources minérales à un niveau sans précédent, avec 70 milliards de tonnes de matières extraites du sous-sol par an et un niveau de consommation desdites ressources par habitant jamais observé jusque-là (Graedel et Cao, 2010 ; Graedel, 2011 ; Wiedmann et al., 2015 ; Elshkaki et al., 2016, 2018). Les prévisions de consommation future sont dans la tendance des évolutions passées, et les quantités annuelles de métaux devant être produites d'ici à 2050 pourraient atteindre 3 à 10 fois les niveaux actuels (Graedel, 2011 ; Graedel et Cao, 2010). La quantité cumulée de métaux à produire au cours des trente-cinq prochaines années dépasserait alors la quantité cumulée produite depuis l'antiquité jusqu'à aujourd'hui. Les enjeux liés aux ressources minérales sont donc énormes, avec des implications de premier ordre dans tous les secteurs économiques et technologiques, dont, en particulier, celui de l'énergie. La présente contribution représente un survol de la question, en insistant sur le caractère multi-facteurs et dynamique des évolutions constatées.
\end{abstract}

\section{Les tendances de la consommation de ressources minérales}

L'émergence économique d'un pays se caractérise par la construction d'infrastructures industrielles et de production, de transformation et d'utilisation de l'énergie, de transport et de communication, de bâtiments... Cette phase de développement consomme principalement des matières premières de base produites en des quantités mondiales supérieures à un million de tonnes par an, telles que le sable et les granulats (50 000 millions de tonnes par an (Mt/an)), le béton (4 $600 \mathrm{Mt} / \mathrm{an}$ ), l'acier et le fer (1 $600 \mathrm{Mt} / \mathrm{an}$, dont la moitié est consommée par la Chine), l'aluminium (60 Mt/an), le cuivre (20 Mt/an), le manganèse, le zinc, le chrome, le plomb, le titane et le nickel. Après une période de forte croissance, la consommation annuelle se stabilise (Bleischwitz et Nechifor, 2016), lorsque le produit intérieur brut (PIB) par habitant avoisine 15000 à 20000 US\$ par habitant. De nombreux pays fortement peuplés comme la Chine, I'Indonésie, l'Inde, le Pakistan et de nombreux pays africains affichent actuellement un $\mathrm{PIB} /$ hab inférieur à ces valeurs. Leur industrialisation sera inévitablement associée à une forte augmentation de la consommation de matières premières, comme cela a été le cas depuis la fin des années 1990 avec l'émergence économique rapide de la Chine.
La demande future en ressources minérales peut être estimée de manière agrégée ou par technologies. L'approche technologique (champs de couleur des Figures 1 et 2 de la page suivante) est basée sur le rythme de construction et de remplacement des technologies avec une intensité matière connue (en t/machine, ou t/puissance électrique installée, etc.). Cette approche nécessite de prendre en compte la durée de vie de chaque type de machines installées, ainsi que le taux de recyclage de chaque métal présent dans chacune de celles-ci, quand elles arrivent en fin de vie. Ce taux de recyclage est d'importance cruciale, car tout métal non recyclé est perdu, et doit donc être compensé par une même quantité de métal primaire. C'est pour cette raison que toutes les estimations ne considérant que l'infrastructure en place sous-estiment largement (dans le cas des métaux faiblement recyclés) les besoins en métaux primaires. Dans le cas de l'énergie, la vitesse de déploiement des technologies est donnée par des scénarios à horizon 2050, qui peuvent être nationaux ou mondiaux.

L'approche agrégée (lignes de la Figure 1) est, quant à elle, basée sur des relations consommation/hab vs $\mathrm{PIB} /$ hab, qui sont contraintes par les évolutions historiques des pays riches depuis les années 1950. Elle est beaucoup plus simple, mais ne permet pas de prendre en compte les nouvelles technologies qui n'existaient pas 
$\mathrm{Fe}$
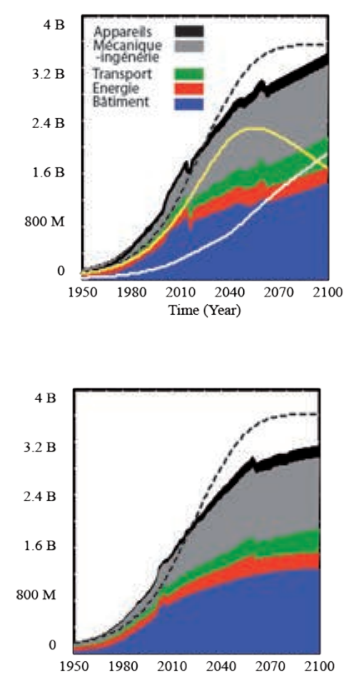

$\mathrm{Al}$
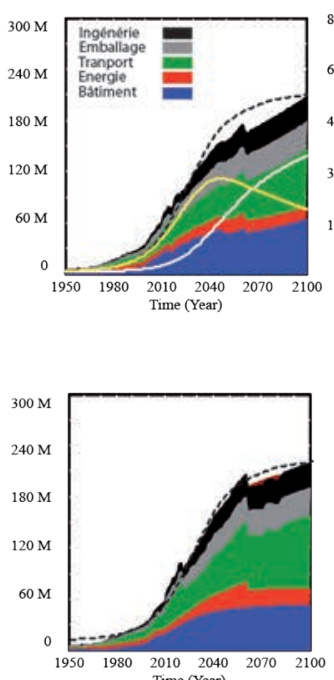

$\mathrm{Cu}$
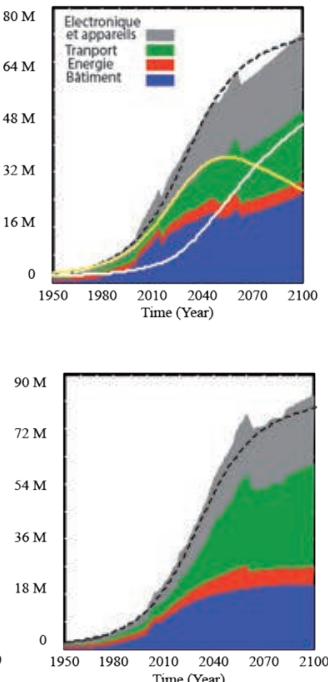

$\mathrm{Ni}$
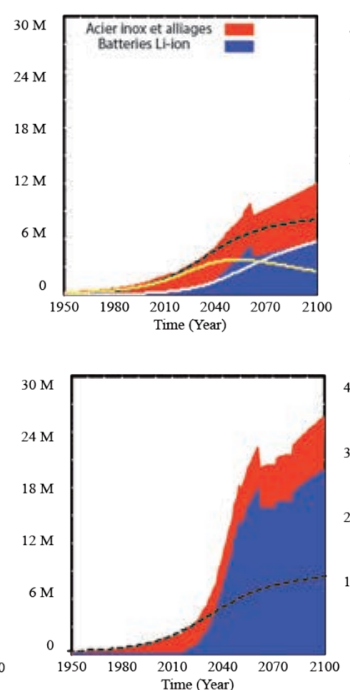

$\mathrm{Li}$
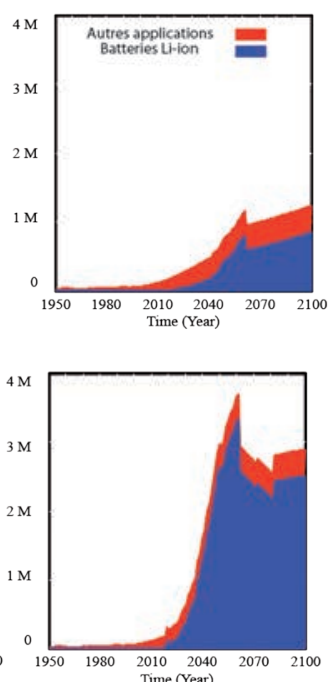

Figure 1 : Besoins annuels au niveau mondial estimés par secteurs (champs de couleur) ou à partir d'évolutions supposées du PIB et de la population (courbe en pointillé : total ; courbe jaune : production primaire ; et courbe blanche : recyclage). Les figures de la première ligne correspondent à un scénario tendanciel (RTS de l'Agence internationale de l'énergie), la deuxième ligne à un scénario visant à réduire les émissions de $\mathrm{CO}_{2}$ pour rester sous les $2^{\circ} \mathrm{C}$ de réchauffement global (B2DS de l'AIE) (source : auteur à partir de son modèle DyMEMDS).

$\mathrm{Fe}$
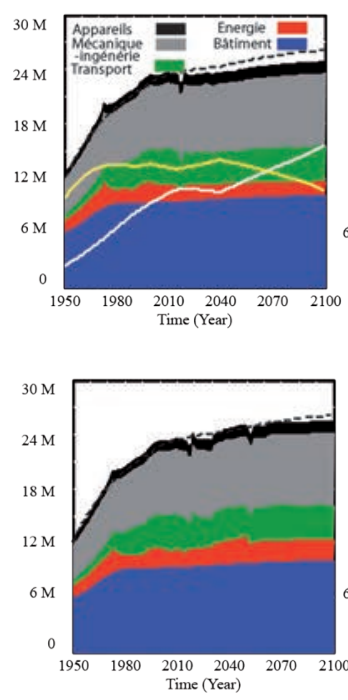

$\mathrm{Al}$
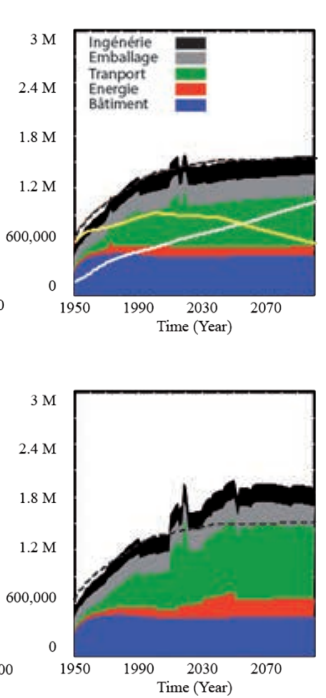

$\mathrm{Cu}$
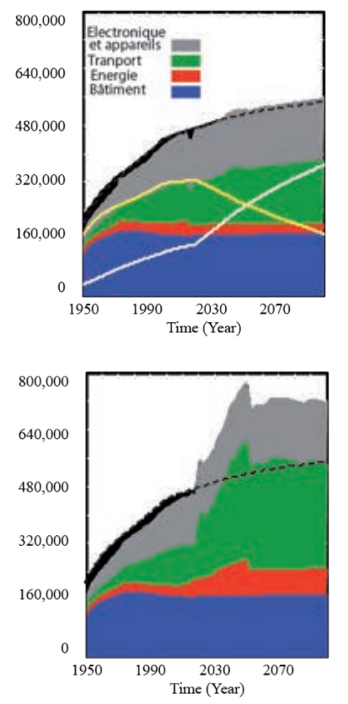

$\mathrm{Ni}$
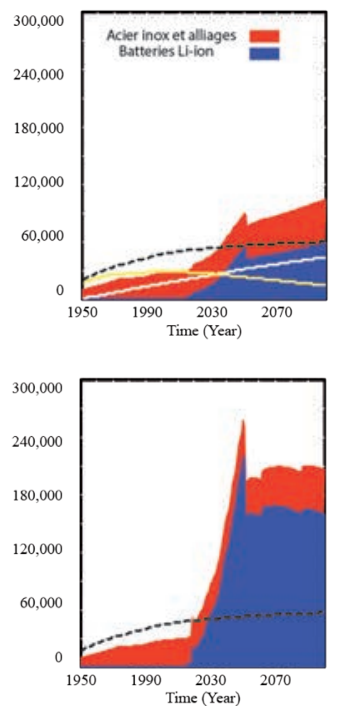

$\mathrm{Li}$
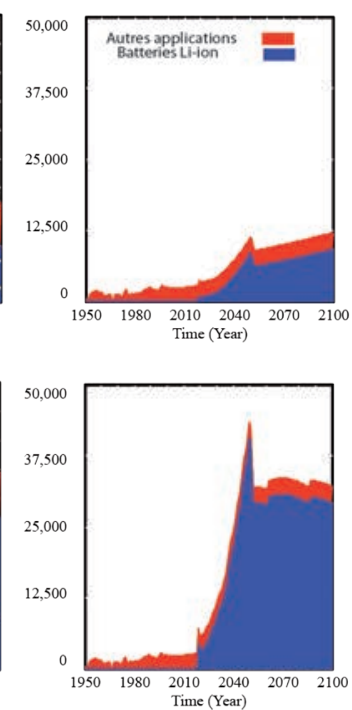

Figure 2 : Besoins annuels estimés pour la France. Même légende que pour la Figure 1. La première ligne correspond au scénario français de référence, la deuxième au scénario Vision90 de l'ADEME (90\% d'énergie renouvelable en 2050) (source : auteur à partir de son modèle DyMEMDS).

ou étaient peu développées il y a trente ans. Cette approche ne s'adresse donc qu'aux matières premières de base utilisées pour des applications classiques, mais en aucun cas aux petits métaux présents dans les nouvelles technologies.

Le modèle dynamique DyMEMDS (Dynamic Modelling of Energy and Matter Demand and Supply), développé dans le cadre du projet SURFER ${ }^{(1)}$, permet d'estimer les futurs

(1) Le projet SURFER, financé par l'ADEME, évalue les matières premières nécessaires pour mener à bien la transition énergétique en France et au niveau mondial pour des scénarios d'une transition forte vers les renouvelables ou de statu quo. besoins en matières grâce aux deux approches précitées et pour des scénarios énergétiques mondiaux ou nationaux. L'évolution mondiale de la demande par rapport à une progression médiane de la population (11 milliards d'habitants en 2100 selon les Nations Unies) et du PIB (données de la Banque mondiale), et l'évolution résultant de scénarios conservateurs ou permettant de limiter le réchauffement en dessous des $2^{\circ} \mathrm{C}$ (ETP-RTS et -B2DS de I'IEA) sont illustrées dans la Figure 1 ci-dessus. Les résultats indiquent une poursuite des évolutions historiques : ainsi, les consommations annuelles mondiales de $\mathrm{Fe}, \mathrm{Al}$, $\mathrm{Cu}$, Ni et Li devraient au minimum doubler, voire tripler d'ici à 2050. Après cette date, la croissance annuelle de 
cette consommation diminue avec l'augmentation du PIB/ hab moyen, et le métal recyclé devient la première source de «production » de Fer, Al et $\mathrm{Cu}$. La production primaire devrait alors décliner.

Les deux types d'estimations soit 1) basées sur les évolutions anticipées de la population et l'évolution logistique de la consommation/hab en fonction de PIB/hab, soit 2) établies par grands secteurs technologiques pour des scénarios de consommation d'énergie donnés, donnent des résultats similaires pour l'acier, le cuivre et l'aluminium. Les utilisations futures de ces matières ne seront en effet pas très différentes de celles passées, même si le développement des technologies électriques, dont le numérique inclus dans les technologies « électroniques et appareils ", et celles remplaçant les carburants fossiles (énergies renouvelables et véhicules électriques), s'accompagne d'un accroissement de la consommation de cuivre pour les scénarios présentant une forte incorporation d'énergie renouvelable (B2DS). Les besoins en cuivre et, dans une moindre mesure, en aluminium sont clairement plus élevés dans le scénario B2DS que dans le scénario RTS, alors que la consommation d'énergie totale (360 PJ, en 2060) est plus faible que dans le scénario de référence RTS (450 PJ). Cela dit, les valeurs estimées restent pour les deux scénarios précités raisonnablement en accord avec les estimations agrégées basées sur les évolutions de PIB et de population. La situation est clairement différente pour le nickel et le lithium, dont les utilisations futures seront différentes de celles observées dans le passé. Ces deux éléments sont utilisés dans les batteries Li-ion qui n'existaient pas il y a trente ans. La pénétration de ces technologies est naturellement plus forte dans les scénarios à prédominance électrique. Pour le Scénario B2DS, les consommations futures de $\mathrm{Li}$ et $\mathrm{Ni}$ sont largement supérieures à celles estimées sur la base des relations consommation-PIB-population. II convient cependant d'être prudent, car les technologies des batteries actuelles ne sont probablement pas celles qui seront utilisées dans vingt ou trente ans. Pour ces raisons, il reste extrêmement difficile d'avoir une estimation fiable des besoins futurs. Ces besoins apparaissent astronomiques au regard des capacités de production actuelles, mais ils ne résultent que de projections à technologie constante. À l'inverse, les besoins estimés pour le cuivre, l'acier et l'aluminium sont beaucoup plus robustes.

Des tendances similaires sont observées au niveau français (voir la Figure 2 de la page précédente), à la différence près que nous avons déjà atteint le niveau de saturation pour l'acier, le cuivre et l'aluminium. Les consommations annuelles de ces matières sont donc relativement stables dans le scénario de référence (première ligne de la Figure 2). Elles sont significativement supérieures dans le cas du cuivre et de l'aluminium pour les scénarios à forte incorporation d'énergies renouvelables (par exemple, Vision90 de l'ADEME, deuxième ligne de la Figure 2). Cela s'explique, d'une part, par la pénétration plus importante des technologies de production d'électricité solaire et d'origine éolienne, qui présentent une intensité matière ( $\mathrm{t} / \mathrm{MW}$ ) plus importante que les centrales nucléaires, et, d'autre part, par la pénétration plus importante des véhicules électriques et hybrides rechargeables, qui ont également des intensités cuivre plus importantes que celles des véhicules thermiques.

Les Figures 1 et 2 indiquent qu'en addition des besoins accompagnant l'émergence économique, la transition vers un monde bas-carbone et l'explosion des technologies numériques constituent des sources importantes de surconsommation par rapport aux tendances historiques. Alors que la croissance de la consommation depuis 1990 a progressé globalement de 1,9\%/an, celle des technologies de l'information et de la communication (TIC) a été de $13 \% / a n$. Ce secteur est grand consommateur de substances rares et de grande pureté ( $\mathrm{Cu}, \mathrm{In}, \mathrm{Ga}, \mathrm{Ge}, \mathrm{Ta}$, $\mathrm{Nb}, \mathrm{Au}, \mathrm{Sb}, \mathrm{Be}, \mathrm{Ag}$, terres rares, etc.) avec des usages dispersifs résultant de la grande dilution dans de nombreux appareils à durée de vie très courte, ce qui limite le potentiel de recyclage et engendre des impacts environnementaux importants. Le cuivre est un métal préoccupant, sa consommation étant dopée par les secteurs de l'énergie et du transport électrique. La Commission européenne vise un objectif, atteindre une part d'énergie renouvelable correspondant au moins à $27 \%$ de la consommation d'énergie de I'UE d'ici à 2030 et les Accord de Paris résultant de la COP21 visent la « neutralité carbone » au niveau mondial au cours de la seconde moitié de ce siècle. Pour atteindre ces objectifs, de grandes infrastructures de production, de transport et de stockage de l'énergie doivent être construites ; or, celles-ci affichent des intensités matières nettement supérieures à celles des technologies conventionnelles utilisant des combustibles fossiles (Vidal et al., 2013, 2017, 2018 ; Hertwich et al., 2014). Actuellement, 1,2 milliard de véhicules légers sont en circulation au niveau mondial ; il est prévu un doublement de ce parc automobile d'ici à 2050. Un véhicule thermique standard contient environ 10 à $20 \mathrm{~kg}$ de cuivre, une voiture électrique en contient trois à quatre fois plus. II faudra donc selon les scénarios entre 200 et $400 \mathrm{Mt}$ de cuivre au niveau mondial, soit l'équivalent de 10 à 20 années de production actuelle pour satisfaire les besoins uniquement de la production et du transport/distribution de l'électricité, ainsi que du transport de marchandises et passagers ; les scénarios intégrant une forte part de renouvelables s'avèrent les plus consommateurs.

\section{Le niveau futur de production permettra-t-il de satisfaire la demande?}

Plusieurs études suggèrent que l'offre future de matières premières ne pourra pas suivre la demande et que la production de plusieurs métaux a déjà atteint son maximum ou atteindra son maximum dans un avenir proche (Hubbert, 1956 ; Meadows et al., 1972 ; Bardi et Lavacci, 2009 ; Laherrère, 2010 ; Kerr, 2014 ; Sverdrup et Ragnasdottir, 2014 ; Northey et al., 2014). Ces travaux font l'hypothèse que la demande et la production augmenteront à un rythme exponentiel, puis s'effondreront lorsque le stock de ressources récupérables sera épuisé. En fait, dans la réalité, la demande se stabilise quand les pays atteignent 
un niveau de PIB/hab > 20000 US\$/hab (voir les Figures 1 et 2). La théorie du pic de Hubbert (Hubbert, 1956) suppose, en outre, que les ressources soient finies et quantifiables, alors que les métaux et minéraux ne sont exploités aujourd'hui que sur une profondeur correspondant à une petite fraction enrichie de la croûte continentale. Si l'amélioration technologique permettait d'exploiter des fractions moins riches ou moins accessibles (plus profondes, en mer), le stock de métaux disponible serait largement supérieur à celui estimé aujourd'hui (2). Enfin, les méthodes empiriques de type Hubbert omettent le recyclage et les couplages économiques. Elles ne peuvent donc se prévaloir d'aucune valeur prédictive sérieuse.

Outre le caractère dynamique de la demande et des réserves, deux autres points doivent être pris en considération pour appréhender l'évolution future de la production de métaux : le coût énergétique de la production et le recyclage. L'énergie ainsi que le coût d'extraction et le prix à long terme des métaux varient en fonction d'une loi de puissance de la dilution qui est inversement proportionnelle à la concentration du métal exploité dans les minerais (Phillips et Edwards, 1976 ; Johnson et al., 2007 ; Gutowski et al., 2013 ; Vidal et al., 2018, 2019, 2020). Une dépendance du prix à la loi de puissance de la dilution signifie qu'à technologie constante, la baisse observée au cours du temps de la teneur des gisements implique une croissance exponentielle de l'énergie nécessaire à la production (Mudd, 2010 ; Norgate et Jahanshahi, 2010). Pourtant, les données historiques ne soutiennent pas ce point (Mariscal et Powell, 2014), car, jusqu'à présent, l'augmentation de l'énergie de production due à la chute de la teneur des minerais a été compensée par l'amélioration de l'efficacité énergétique et de la productivité, qui elles-mêmes sont les fruits de l'amélioration technologique (Yellishetty et al., 2010 ; Gutowski et al., 2013). L'amélioration technologique explique pourquoi les quantités produites de métaux et le volume de leurs réserves ont tous deux suivi une croissance quasi exponentielle et enregistré une décroissance de leur prix (en monnaie constante) entre 1900 et 2000 . Mais là encore, il convient d'être prudent, car cette tendance n'est pas pérenne. En effet, le gain en énergie devient de plus en plus faible avec le temps, alors que la quantité d'énergie supplémentaire nécessaire pour extraire des métaux de moins en moins concentrés dans les gisements augmente. II existe ainsi un point critique au-delà duquel le gain technologique ne compense plus la perte résultant de la dégradation de la qualité des ressources, la production demandant en effet de plus en plus d'énergie. Pour beaucoup de métaux, ce point est déjà atteint ou il le sera avant 2050. L'augmentation de prix entraîne mécaniquement une baisse de la demande et de la production primaire, qui est renforcée par le recyclage massif quand le niveau de saturation est atteint (voir la Figure 1). Cette analyse rapide nous amène à la conclusion que les grandes tendances passées, à savoir une augmentation des réserves et une diminution des coûts de production et

(2) Cette hypothèse néglige naturellement des impacts environnementaux pourtant décuplés par l'extraction de ressources diluées et difficilement accessibles. du prix sous l'effet de l'amélioration technologique, ne sauraient se poursuivre indéfiniment si nous dépendons d'un stock fini d'énergie, à savoir celui des hydrocarbures. Si nous pouvions utiliser à l'avenir des sources d'énergies renouvelables « illimitées » à un prix raisonnable, la question se poserait différemment.

Le recyclage est également un paramètre à prendre en compte. Contrairement aux combustibles fossiles, les métaux primaires ne sont pas perdus après utilisation, les produits métalliques fabriqués aujourd'hui constituant les réserves du recyclage de demain. L'augmentation de la part du recyclage ne suffit pas pour répondre à la demande en période de croissance, car nous ne pouvons recycler qu'une partie des biens de consommation et des équipements créés il y a plusieurs décennies, à une période où la production de matières premières était inférieure aux besoins actuels. Cependant, lorsque la plupart des pays auront atteint un PIB/habitant correspondant au niveau de saturation, le recyclage des stocks de métaux présents dans les équipements de notre société actuelle pourraient en théorie devenir la principale source de matières premières (voir la Figure 1, pour le cuivre). En pratique, le potentiel de recyclage est limité par des facteurs économiques, tels que la différence entre le prix des métaux primaires et le coût de leur recyclage. Seuls les métaux présents à des concentrations suffisamment élevées dans les produits en fin de vie pourront être recyclés, car le coût de leur recyclage et le prix des énergies nécessaires à la production des métaux les plus dilués restent non compétitifs par rapport aux coûts de leur production primaire (Johnson et al., 2007 ; Vidal et al., 2017). Cela explique pourquoi la plupart des métaux rares utilisés dans les hautes technologies ne sont pas recyclés aujourd'hui. Une augmentation future des prix de ces métaux favorisera leur recyclage, ce qui est une bonne nouvelle. Pour autant, le recyclage reste actuellement basé sur une disponibilité de flux (production annuelle de déchets) plutôt que de stock. C'est là une des différences majeures par rapport à la production primaire qui, elle, utilise des réserves (un stock). S'il existe un risque que le flux de déchets s'épuise, la filière de recyclage deviendra rapidement non viable. C'est une des raisons pour lesquelles il est vital de concevoir les produits de manière à faciliter leur recyclage après utilisation, même si cela se fait aux dépens de la performance ou du prix.

\section{Références}

BARDI U. \& LAVACCHI A. (2009), "A Simple interpretation of Hubbert's model of resource depletion", Énergies, 2(3), pp. 646-661, doi:10.3390/en20300646

BARDI U. \& PAGANI M. (2008), "Peak Minerals", The Oil Drum, http://www.theoildrum.com/ node/3086

BLEISCHWITZ R. \& NECHIFOR V. (2016), "Saturation and Growth Over Time: When Demand for Minerals Peaks", Prisme 34, doi:10.13140/RG.2.2.24146.15049, https://www.centre-cournot. org/img/pdf/prisme_fr/Prisme $\% 20 N^{\circ} 34 \% 20$ Novembre $\% 20$ 2016\%20(english).pdf

ELSHKAKI A., GRAEDEL T. E., CIACCI L. \& RECK B. K. (2018), "Resource Demand Scenarios for the Major Metals", Environmental Science \& Technology 52 (5), pp. 2491-2497. 
ELSHKAKI A., GRAEDEL T. E., CIACCI L. \& RECK B. K. (2016), "Copper demand, supply, and associated energy use to 2050", Global environmental change 39, pp. 305-315.

GRAEDEL T. E. (2011), "On the Future Availability of the Energy Metals", Annual Review of Materials Science 41, pp. 323-335.

GRAEDEL T. E. \& CAO J. (2010), "Metal spectra as indicators of development", Proceedings of the National Academy of Sciences 107, pp. 20905-20910.

GUTOWSKI T. G., SAHNI S., ALLWOOD J. M., ASHBY M. F. \& WORRELL E. (2013), The energy required to produce materials: constraints on energy-intensity improvements, parameters of demand, Philosophical Transactions of the Royal Society, A 371: 20120003

HERTWICH E. G., GIBON T., BOUMAN E. A., ARVESEN A., SUH S., HEATH G. A., BERGESEN J. D., RAMIREZ A., VEGA M. I. \& SHI L. (2015), "Integrated life-cycle assessment of electricity-supply scenarios confirms global environmental benefit of low-carbon technologies", Proceedings of the National Academy of Sciences 112, pp. 6277-6282.

HUBBERT M. K. (1956), "Nuclear Energy and Fossil Fuels", Drilling and production practice 23, pp. 7-25.

JOHNSON J., HARPER E. M., LIFSET R. \& GRAEDEL T. E. (2007), "Dining at the periodic table: metal concentrations as they relate to recycling", Environmental Science \& Technology 41, pp. 17591765.

KERR R. A. (2014), "The coming copper peak", Science 343, pp. 722-724.

LAHERRERE J. (2010), posted by de SOUSA L., Copper peak. The oildrum Europe, April, 1.2010;6307, pp. 1-27, http://europe. theoildrum.com/node/6307

MARISCAL R. \& POWELL A. (2014), "Commodity Price Booms and Breaks: Detection, Magnitude and Implications for Developing Countries", Inter-American Development Bank, Working Paper $n^{\circ} 444$, January.
MUDD G. (2010), "The environmental sustainability of mining in Australia: key mega-trends and looming constraints", Resources Policy 35, pp. 98-115.

NORGATE T. \& JAHANSHAHI S. (2010), "Low grade ores. Smelt, leach or concentrate?”, Minerals Engineering 23, pp. 65-73.

NORTHEY S., MOHR S., MUDD G. M., WENG Z. \& GIURCO D. (2014), "Modelling future copper ore grade decline based on a detailed assessment of copper resources and mining", Resources, Conservation and Recycling 83, pp. 190-211.

SVERDRUP H. U. \& RAGNASDOTTIR K. A. (2014), "Natural resources in a planetary perspective", Geochemical perspectives 3, pp. 129-341.

VIDAL O. (2018), Matières premières et énergie, les enjeux de demain, ISTE Editions Ltd, $245 \mathrm{p}$.

VIDAL O., GOFFÉ B. \& ARNDT N. (2013), "Metals for a low-carbon society", Nature Geoscience 6, pp. 894-896.

VIDAL O., ROSTOM F., FRANCOIS C. \& GIRAUD G. (2017), "Global trends in metal consumption and supply: the raw material-energy nexus", Elements 13(5), pp. 319-324.

VIDAL O., ROSTOM F., FRANÇOIS C. \& GIRAUD G. (2019), "Prey-predator long-term modeling of copper reserves, production, recycling, price, and cost of production", Environmental Science \& Technology 53(19), pp. 11323-11336.

VIDAL O., LE BOULZEC H. \& FRANÇOIS C. (2018), "Modelling the material and energy cost of the transition to low-carbon energy", EPJ Web of Conferences 189, 00018, https://doi.org/10.1051/ epjconf/201818900018

WIEDMANN T. O., SCHANDL H., LENZEN M., MORAN D., SUH S., WEST J. \& KANEMOTO K. (2015), "The material footprint of nations", Proceedings of the National Academy of Sciences 112, pp. 6271-6276.

YELLISHETY M., RANJITH P. G. \& THARUMARAJAH A. (2010), Ressources, Conservation and Recycling 54, pp. 1084-1094. 